\title{
Singular perturbation analysis of a reduced model for collective motion: A renormalization group approach
}

\author{
Chiu Fan Lef* \\ Max Planck Institute for the Physics of Complex Systems, Nöthnitzer Str. 38, 01187 Dresden, Germany
}

(Dated: October 27, 2018)

\begin{abstract}
In a system of noisy self-propelled particles with interactions that favor directional alignment, collective motion will appear if the density of particles is beyond a critical density. Starting with a reduced model for collective motion, we determine how the critical density depends on the form of the initial perturbation. Specifically, we employ a renormalization-group improved perturbative method to analyze the model equations, and show analytically, up to first order in the perturbation parameter, how the critical density is modified by the strength of the initial angular perturbation in the system.
\end{abstract}

PACS numbers: 05.65.+b, 64.60.-i, 02.30.Mv, 05.40.-a, 45.50.-j

\section{INTRODUCTION}

The interesting phenomena of flocking in animals 1 5] and self-organized patterns in motile cells [ 6 - 8$]$ are currently driving the intense theoretical study of collective motion among self-propelled particles [9 21]. Models for collective motion usually involve motile particles that possess alignment interactions and angular noise. Collective motion is then observed if the density of particles increases beyond a certain threshold. We have previously argued that besides density fluctuations, the initial fluctuations in the heading directions of the particles constitute another important aspect of the system [22]. Here, we determine the critical density as a function of the initial perturbation strength by analyzing a reduced model for collective motion. Specifically, we assume that the angular noise strength, $\epsilon$, is small and employ it as the perturbation parameter. We then find that the solution obtained by the naive perturbation method is plagued by divergences due to the appearances of temporal secular terms [23, 24], which we subsequently eliminate up to order $\epsilon$ by the renormalization group method [25 30$]$.

\section{MODEL}

In this work, we consider a minimal model for collective motion in two dimensions introduced in 20]. In this model, every particle is assumed to have constant speed $u$, and that their interactions consist only of a directional alignment mechanism, with interaction strength $\epsilon \gamma$. Noise, of strength $\epsilon$, is incorporated in the direction of travel. Note that the factor $\epsilon$ in front of the interaction strength is to emphasize that our perturbative treatment will be on both the interaction and noise strengths. Since $\gamma$ is still a free parameter, these two effects can be varied independently.

*Electronic address: cflee@pks.mpg.de
FIG. 1: (a) The form of the initial perturbation with $b=0.1$ and $\eta=0.5$. (b) The form of the function $R(x)$ (c.f. Eq. (22)). (c) \& (d) The temporal evolutions of $\alpha$ and $\beta$, with $\epsilon=0.5$ and $\gamma=0.9$, respectively. These results are obtained by performing numerical integrations of Eqs (1) with the initial square wave perturbation approximated by the function: $b[\tanh (30(x+\eta))-\tanh (30(x-\eta))]$.
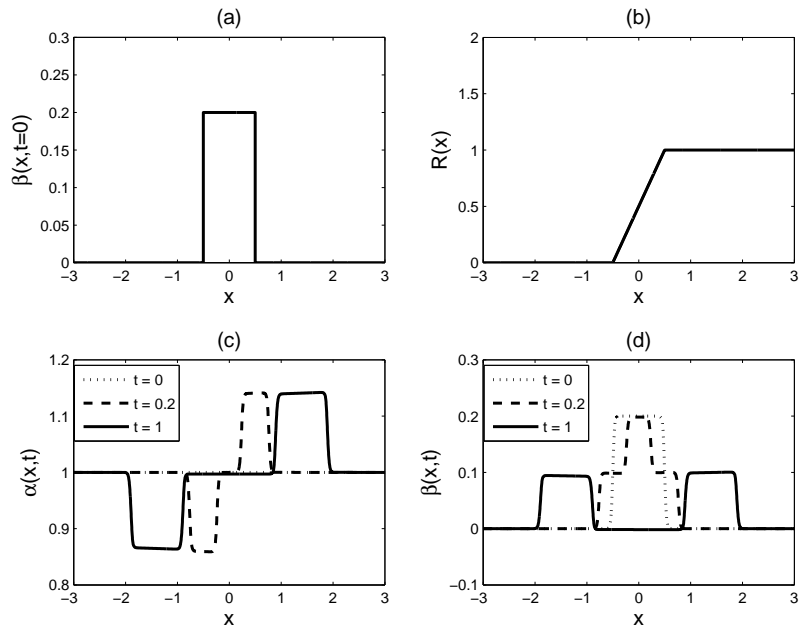

Starting from the Fokker-Planck equation describing this model, we have previously argued that at the onset of collective motion, the model equations can be approximated by a finite set of coupled partial differential equations (PDEs) 22]. Here, we will study the simplest set of those coupled PDEs, which corresponds to the following two coupled PDEs:

$$
\partial_{t} \alpha=-2 u \partial_{x} \beta \quad, \quad \partial_{t} \beta=-u \partial_{x} \alpha+\epsilon(\gamma \alpha \beta-\beta) .
$$

In the above equations, $\alpha$ corresponds to the local particle density, and $\beta$ corresponds to the local vectorial order parameter of the system, i.e., a non-zero $\beta$ implies the existence of collective motion. Note that the problem is 
reduced to one dimension as we assume that the initial directional preference of the system is along the $x$-axis.

The derivation of and the approximations involved in the model equations are elaborated in 22] and we summarize the essential steps in Appendix A for completeness. We are primarily interested in the regime where $\epsilon \ll u$ and $\gamma \sim \mathcal{O}(1)$. The first condition allows us to study the reduced model perturbatively with respect to $\epsilon$, and the second condition is our main interest because it is the region where the transition from disordered motion to collective motion occurs, as we will see shortly.

At the mean field level, i.e., if the spatial variations in $\alpha$ and $\beta$ are ignored, the threshold for collective motion is (c.f. Eqs (1)) 20, 22]:

$$
\rho_{c}=\gamma^{-1}
$$

where $\rho_{c}$ denotes the critical density. We have previously argued that such a mean-field picture is incomplete because the initial perturbation to the system should play a major role as well [22]. For instance, let us assume that the initial density is $\rho$, i.e., $\alpha(x)=\rho$, and $\gamma<\rho^{-1}$. In other words, no collective motion would be expected according to the mean-field description. Let us now consider an initial perturbation in the form of a square wave in $\beta$ with magnitude $2 b$. As shown in the next section, such a perturbation would induce a density wave of magnitude $\sqrt{2} b$ in $\alpha$ that travels in the positive $x$ direction (c.f. Fig. 1(c) and (d)). Within the traveling density wave, $\alpha \sim \rho+\sqrt{2} b$. If

$$
\rho+\sqrt{2} b>\gamma^{-1},
$$

then the density within the traveling wave is beyond the collective motion threshold. Therefore, according to the mean-field criterion, we would naively expect that such a density wave will be amplified, and thus signals the onset of collective motion. In other words, the critical density may depend on the strength of the initial perturbation perturbation. In the next two sections, we will verify this expectation by analyzing the reduced model perturbatively.

\section{NAIVE PERTURBATIVE TREATMENT}

We are concerned with the progression of a perturbation to an initially disordered system, and so we are primarily interested in the initial conditions of the form:

$$
\alpha(x, t=0)=A \quad, \quad \beta(x, t=0)=2 f(x),
$$

where $A$ corresponds to the local density and $f$ is an arbitrary function that describes the initial perturbation in the angular directions of the particles.

We will now consider the model perturbatively by making the following expansions for $\alpha$ and $\beta$ :

$$
\begin{aligned}
& \alpha=\alpha_{0}+\epsilon \alpha_{1}+\mathcal{O}\left(\epsilon^{2}\right) \\
& \beta=\beta_{0}+\epsilon \beta_{1}+\mathcal{O}\left(\epsilon^{2}\right) .
\end{aligned}
$$

To the zeroth order, Eqs (1) give

$$
\partial_{t} \alpha_{0}=-2 u \partial_{x} \beta_{0} \quad, \quad \partial_{t} \beta_{0}=-u \partial_{x} \alpha_{0},
$$

and the solutions are the standard traveling wave equations:

$$
\begin{aligned}
\alpha_{0}(x, t) & =A+\sqrt{2}[f(x-\sqrt{2} u t)-f(x+\sqrt{2} u t)] \\
\beta_{0}(x, t) & =f(x-\sqrt{2} u t)+f(x+\sqrt{2} u t)
\end{aligned}
$$

Note that since the units of time and length can be defined arbitrarily, we will use these degrees of freedom to set $u=1 / \sqrt{2}$ and $A=1$ from now on.

In the first order in $\epsilon$, Eqs (1) lead to

$$
\begin{aligned}
& \partial_{t} \alpha_{1}=-\sqrt{2} \partial_{x} \beta_{1} \\
& \partial_{t} \beta_{1}=-\frac{1}{\sqrt{2}} \partial_{x} \alpha_{1}+\gamma \alpha_{0} \beta_{0}-\beta_{0} .
\end{aligned}
$$

We can eliminate the $\beta_{1}$ term above by combining the partial derivative of Eq. (9) with respect to $t$ and the partial derivative of Eq. (10) with respect to $x$. We then obtain:

$$
\left(\partial_{t}^{2}-\partial_{x}^{2}\right) \alpha_{1}=\sqrt{2} \partial_{x}\left(\beta_{0}-\gamma \alpha_{0} \beta_{0}\right)
$$

The above inhomogeneous wave equation is supplemented by the initial conditions;

$$
\begin{aligned}
\alpha_{1}(x, t=0) & =0 \\
\partial_{t} \alpha_{1}(x, t=0) & =-\sqrt{2} \partial_{x} \beta_{1}(x, t=0)=0
\end{aligned}
$$

where the second equality in Eq. (13) follows from Eq. (9) and the fact that $\beta_{1}(x, t=0)=0$. The general solution for $\alpha_{1}$ can thus be written as (e.g., see ch. 8.2 in [31]):

$$
\alpha_{1}=\frac{1}{\sqrt{2}} \int_{0}^{t} \int_{x-\left(t-t^{\prime}\right)}^{x+\left(t-t^{\prime}\right)}\left[\partial_{x^{\prime}}\left(\beta_{0}-\gamma \alpha_{0} \beta_{0}\right)\right] \mathrm{d} x^{\prime} \mathrm{d} t^{\prime} .
$$

By plugging the expressions for $\alpha_{0}$ and $\beta_{0}$ (c.f. Eqs (8) ) into the above integral, we find

$$
\begin{aligned}
\alpha_{1}= & \frac{t}{\sqrt{2}}\left[(\gamma-1)\left(f_{-}-f_{+}\right)+\gamma\left(f_{-}^{2}+f_{+}^{2}\right)\right] \\
& +\frac{\gamma}{\sqrt{2}}\left(h_{-}-h_{+}\right),
\end{aligned}
$$

where $f_{ \pm} \equiv f(x \pm t), h_{ \pm} \equiv h(x \pm t)$ and the function $h$ is defined by the following ordinary differential equation (ODE):

$$
\partial_{y} h(y)=f^{2}(y)
$$

Also, we can deduce an expression for $\beta_{1}$ by combining Eq. (15) and Eq. (91):

$$
\begin{aligned}
\beta_{1}= & \frac{t}{2}\left[(\gamma-1)\left(f_{-}+f_{+}\right)+\gamma\left(f_{-}^{2}-f_{+}^{2}\right)\right] \\
& +\frac{1-\gamma}{2}\left(g_{-}-g_{+}\right) .
\end{aligned}
$$

where $g_{ \pm} \equiv g(x \pm t)$ and the function $g$ is defined by the following ODE:

$$
\partial_{y} g(y)=f(y)
$$




\section{Example}

Consider the case where the initial perturbation is a square wave of width $2 \eta$ and of magnitude $2 b$, i.e.,

$$
\beta(x, t=0)=2 b[H(x+\eta)-H(x-\eta)],
$$

where $H(y)$ is the Heaviside function such that $H(y)=1$ for $y \geq 0$, and zero otherwise. Note that in order for the perturbative treatment to be valid, the magnitude $b$ has to satisfy the following conditions: $\epsilon b, \epsilon b^{2} \ll u=1 / \sqrt{2}$ (c.f. Eq. (1) ). For this square wave perturbation, we have from Eqs (16) and (19)

$$
h_{ \pm}=b^{2} R(x \pm t) \quad, \quad g_{ \pm}=b R(x \pm t) .
$$

where (c.f. Fig. 1(b))

$$
R(y)= \begin{cases}0, & y<-\eta \\ y, & -\eta \leq y<\eta \\ 2 \eta, & y \geq \eta\end{cases}
$$

The temporal evolutions of $\alpha$ and $\beta$ as a result of this initial perturbation are shown in Fig. 1(c) and (d).

\section{RENORMALIZATION GROUP TREATMENT}

The appearances of $t$ in front of the terms in squared brackets in Eqs (15) and (17) signify that the perturbative solution only makes sense for $t \ll \epsilon^{-1}$. In other words, the problem is a singular perturbation problem and the terms in squared brackets are called secular terms [24]. To eliminate such secular terms, we will now follow the renormalization group $(\mathrm{RG})$ method introduced in [25, 26].

In the RG method, we first introduce an arbitrary time $\tau$ and split $t$ as $t-\tau+\tau$. We then rewrite $f(x \pm t)$ as $B_{ \pm}(\tau) f_{ \pm}$where $f_{ \pm} \equiv f(x \pm(t-C(\tau))$, such that

$$
\begin{aligned}
& 1=\left[1+\sum_{n=1}^{\infty} b_{n}^{ \pm}(\tau) \epsilon^{n}\right] B_{ \pm}(\tau) \\
& 0=C(\tau)+\sum_{n=1}^{\infty} c_{n}(\tau) \epsilon^{n} .
\end{aligned}
$$

The series $\left\{b_{n}^{ \pm}\right\}$and $\left\{c_{n}\right\}$ are the multiplicative and additive renormalization constants respectively, and they will be chosen order by order in $\epsilon$ to eliminate the terms containing $\tau$.

Applying the above expansions to $\alpha$ and ignoring the non-secular term for the time being, we have for up to order $\epsilon$,

$$
\begin{aligned}
\alpha= & 1+\sqrt{2}\left[\left(1+b_{1}^{-} \epsilon\right) B_{-} f_{-}-\left(1+b_{1}^{+} \epsilon\right) B_{+} f_{+}\right] \\
& +\epsilon(t-\tau)\left[\frac{\gamma-1}{\sqrt{2}}\left(B_{-} f_{-}-B_{+} f_{+}\right)\right.
\end{aligned}
$$

$$
\begin{aligned}
& \left.+\gamma\left(B_{-}^{2} f_{-}^{2}+B_{+}^{2} f_{+}^{2}\right)\right] \\
& +\epsilon \tau\left[\frac{\gamma-1}{\sqrt{2}}\left(B_{-} f_{-}-B_{+} f_{+}\right)+\gamma\left(B_{-}^{2} f_{-}^{2}+B_{+}^{2} f_{+}^{2}\right)\right](28)
\end{aligned}
$$

Our strategy now is to eliminate the term shown in Eq. (28) by picking $b_{1}^{ \pm}$appropriately. We will again focus on the square wave as our initial perturbation, i.e.,

$$
f(y)=H(y+\eta)-H(y-\eta) .
$$

The virtue of this choice is the property that $f_{ \pm}^{2}=f_{ \pm}$. It is due to this critical property that we can perform the elimination simply by defining the following:

$$
b_{1}^{ \pm}=\tau\left[\frac{\gamma-1}{\sqrt{2}} B_{ \pm}+\gamma B_{ \pm}^{2}\right]
$$

With $b_{1}^{ \pm}$defined as above, $\alpha$ is simplified to

$$
\begin{aligned}
\alpha= & 1+\sqrt{2}\left[B_{-} f_{-}-B_{+} f_{+}\right] \\
& +\epsilon(t-\tau)\left[\frac{\gamma-1}{\sqrt{2}}\left(B_{-} f_{-}-B_{+} f_{+}\right)\right. \\
& \left.+\gamma\left(B_{-}^{2} f_{-}^{2}+B_{+}^{2} f_{+}^{2}\right)\right]+\mathcal{O}\left(\epsilon^{2}\right) .
\end{aligned}
$$

The same expansion renders $\beta$ as follows:

$$
\begin{aligned}
\beta= & B_{-} f_{-}+B_{+} f_{+} \\
& +\epsilon(t-\tau)\left[\frac{\gamma-1}{2}\left(B_{-} f_{-}+B_{+} f_{+}\right)\right. \\
& \left.+\frac{\gamma}{\sqrt{2}}\left(B_{-}^{2} f_{-}^{2}-B_{+}^{2} f_{+}^{2}\right)\right]+\mathcal{O}\left(\epsilon^{2}\right) .
\end{aligned}
$$

The benefit of the above exercise is that as $\tau$ is arbitrary, the divergence of the original secular terms can be eliminated by picking $\tau$ to be close to $t$. Now, the next step is to also eliminate $\tau$ in the above equations. As $\alpha$ and $\beta$ should be independent of how $\tau$ is chosen, their derivatives with respect to $\tau$ are zero. Therefore, to first order in $\epsilon$, we arrive at the following differential equations governing $B_{ \pm}$and $C$ :

$$
\begin{aligned}
f_{-} \frac{\partial B_{-}}{\partial \tau}-f_{+} \frac{\partial B_{+}}{\partial \tau}= & \frac{\epsilon}{\sqrt{2}}\left[\gamma\left(B_{-}^{2} f_{-}+B_{+}^{2} f_{+}\right)\right. \\
& \left.+\frac{\gamma-1}{\sqrt{2}}\left(B_{-} f_{-}-B_{+} f_{+}\right)\right] \\
f_{-} \frac{\partial B_{-}}{\partial \tau}+f_{+} \frac{\partial B_{+}}{\partial \tau}= & \frac{\epsilon}{\sqrt{2}}\left[\gamma\left(B_{-}^{2} f_{-}-B_{+}^{2} f_{+}\right)\right. \\
& \left.+\frac{\gamma-1}{\sqrt{2}}\left(B_{-} f_{-}+B_{+} f_{+}\right)\right] \\
\frac{\partial C}{\partial \tau}= & 0 .
\end{aligned}
$$

Eq. (38) indicates that we can simply set $C(\tau)$ to be zero. Furthermore, Eqs (36) and (37) can be decoupled and we 
arrive at the following two ODEs:

$$
\begin{aligned}
& \frac{\partial B_{-}}{\partial \tau}=\frac{\epsilon}{\sqrt{2}}\left[\gamma B_{-}^{2}+\frac{\gamma-1}{\sqrt{2}} B_{-}\right] \\
& \frac{\partial B_{+}}{\partial \tau}=\frac{\epsilon}{\sqrt{2}}\left[-\gamma B_{+}^{2}+\frac{\gamma-1}{\sqrt{2}} B_{+}\right] .
\end{aligned}
$$

The corresponding solutions are

$$
\begin{aligned}
& B_{+}(\tau)=\frac{\gamma-1}{b_{+} \mathrm{e}^{-\epsilon(\gamma-1) \tau / 2}+\sqrt{2} \gamma} \\
& B_{-}(\tau)=\frac{\gamma-1}{b_{-} \mathrm{e}^{-\epsilon(\gamma-1) \tau / 2}-\sqrt{2} \gamma}
\end{aligned}
$$

where $b_{ \pm}$are obtained from the initial condition of the problem.

By picking $\tau=t$, we can finally eliminate the original secular terms, and the expressions for $\alpha$ and $\beta$ become

$$
\begin{aligned}
\alpha & =1+\sqrt{2}\left(B_{-} f_{-}-B_{+} f_{+}\right)+\epsilon \gamma\left(h_{-}-h_{+}\right)+\mathcal{O}\left(\epsilon^{2}\right) \\
\beta & =B_{-} f_{-}+B_{+} f_{+}+\epsilon \frac{1-\gamma}{2}\left(g_{-}-g_{+}\right)+\mathcal{O}\left(\epsilon^{2}\right)
\end{aligned}
$$

Note that in the above solutions, we have reinstalled the non-secular terms $h_{ \pm}$and $g_{ \pm}$, which are defined in Eqs (21). We show that $h_{ \pm}$and $g_{ \pm}$are not modified under the renormalization procedures in Appendix B.

We now consider the temporal evolutions of the system under the designated initial perturbation. By assumption, we have at $t=0$,

$$
\alpha(x, t=0)=1 \quad, \quad \beta(x, t=0)=2 b f(0) .
$$

Hence, $B_{ \pm}(0)=b$ and so

$$
b_{+}=\frac{\gamma-1}{b}-\sqrt{2} \gamma \quad, \quad b_{-}=\frac{\gamma-1}{b}+\sqrt{2} \gamma .
$$

Let us focus only on the density wave in $\alpha$ traveling to the right. The magnitude of the density wave is

$$
B_{-}(t)=\frac{b(\gamma-1)}{((\gamma-1)+\sqrt{2} b \gamma) \mathrm{e}^{-\epsilon(\gamma-1) t / 2}-\sqrt{2} b \gamma} .
$$

If $\gamma>1$, then $B_{-}$grows with time since the first term in the denominator approaches $\sqrt{2} b \gamma$ as $t$ grows [32]. In other words, the original perturbation is amplified, which is a signature for the onset of collective motion. This scenario corresponds exactly to our mean-field prediction (c.f. Eq. (2) ). The more interesting case is that even for $\gamma<1, B_{-}$still grows if

$$
b>\frac{1-\gamma}{\sqrt{2} \gamma} .
$$

This is the condition discussed previously and based purely intuition ground (c.f. Eq. (3) ). In other words, the critical density in the presence of the perturbation considered is shifted downwards and becomes

$$
\rho_{c}=\gamma^{-1}-\sqrt{2} b .
$$

\section{CONCLUSION}

In this paper, we have demonstrated how the critical density for collective motion can depend on the strength of the initial angular perturbation. Our conclusion is based on a renormalization-group improved perturbative treatment of a reduced model for collective motion. At the simulation level, our results suggest that simulations with different implementations of the initial perturbations may lead to different values of the critical density.

\section{Appendix A: Reduced model}

For completeness, we summarize here the essential steps in deriving the equations describing the reduced model studied here [22]. We consider a minimal model for collective motion in two dimensions, where every particle is assumed to have constant speed, $u$, and that their interactions consist only of a directional alignment mechanism. Noise, of strength $\epsilon$, is incorporated in the direction of travel. Specifically, let there be $N$ particles in a volume of $V$, their equations of motion are:

$$
\begin{aligned}
\frac{\mathrm{d} \mathbf{r}_{i}}{\mathrm{~d} t} & =\frac{2 u}{\pi} \mathbf{v}\left(\theta_{i}\right) \\
\frac{\mathrm{d} \theta_{i}}{\mathrm{~d} t} & =-\frac{\partial U(\mathbf{R}, \Theta)}{\partial \theta_{i}}+\sqrt{2 \epsilon} \eta_{i}(t)
\end{aligned}
$$

where $1 \leq i \leq N, \mathbf{R} \equiv\left(\mathbf{r}_{1}, \ldots, \mathbf{r}_{N}\right), \Theta \equiv\left(\theta_{1}, \ldots, \theta_{N}\right)$, $\mathbf{v}(\theta) \equiv(\cos \theta, \sin \theta)$, and the noise is assumed to be Gaussian characterized by the following moments:

$$
\left\langle\eta_{i}(t)\right\rangle=0 \quad, \quad\left\langle\eta_{i}(t) \eta_{j}\left(t^{\prime}\right)\right\rangle=\delta_{i j} \delta\left(t-t^{\prime}\right) .
$$

Moreover, the alignment interaction is assumed to be of very short range and can thus be approximated by a delta function:

$$
U(\mathbf{R}, \Theta)=-\frac{\epsilon \gamma}{\pi} \sum_{i<j} \delta^{(2)}\left(\mathbf{r}_{i}-\mathbf{r}_{j}\right) \cos \left(\theta_{i}-\theta_{j}\right)
$$

where $\epsilon \gamma$ corresponds to the coupling strength. If we denote the probability distribution of the density of particles in the state $(\mathbf{R}, \Theta)$ at time $t$ by $f(t, \mathbf{R}, \Theta)$, then the Fokker-Planck equation corresponding to the system is [33]:

$$
\begin{aligned}
\frac{\partial f}{\partial t}= & \sum_{i}\left\{\epsilon \frac{\partial^{2}}{\partial \theta_{i}^{2}} f-\frac{2 u}{\pi} \nabla_{\mathbf{r}_{i}} \cdot\left[\mathbf{v}\left(\theta_{i}\right) f\right]\right\} \\
& +\frac{\epsilon \gamma}{\pi} \sum_{i<j} \frac{\partial}{\partial \theta_{i}}\left[\delta^{(2)}\left(\mathbf{r}_{i}-\mathbf{r}_{j}\right) \sin \left(\theta_{i}-\theta_{j}\right) f\right]
\end{aligned}
$$

Focusing now on the single-particle density function, $\rho$, where

$$
\rho\left(\mathbf{r}_{1}, \theta_{1}\right)=\frac{(N !) \int \mathrm{d} r_{2} \cdots \mathrm{d} r_{N} \mathrm{~d} \theta_{2} \cdots \mathrm{d} \theta_{N} f(\mathbf{R}, \Theta)}{(N-1) !}
$$


Eq. (A5) becomes

$$
\begin{aligned}
\frac{\partial \rho(\mathbf{r}, \theta)}{\partial t}= & \epsilon \frac{\partial^{2} \rho(\mathbf{r}, \theta)}{\partial \theta^{2}}-\frac{2 u}{\pi}\left[\cos \theta \frac{\partial \rho(\mathbf{r}, \theta)}{\partial x}+\sin \theta \frac{\partial \rho(\mathbf{r}, \theta)}{\partial y}\right] \\
& +\frac{\epsilon \gamma}{\pi} \frac{\partial}{\partial \theta}\left[\int \mathrm{d} \theta^{\prime} \sin \left(\theta-\theta^{\prime}\right) \rho^{(2)}\left(\mathbf{r}, \theta, \mathbf{r}, \theta^{\prime}\right)\right] .(\mathrm{A} 7)
\end{aligned}
$$

where

$\rho^{(2)}\left(\mathbf{r}_{1}, \theta_{1}, \mathbf{r}_{2}, \theta_{2}\right)=\frac{(N !) \int \mathrm{d} r_{3} \cdots \mathrm{d} r_{N} \mathrm{~d} \theta_{3} \cdots \mathrm{d} \theta_{N} f(\mathbf{R}, \Theta)}{(N-2) !}$.

We now employ the molecular chaos assumption to close the above hierarchical equation by approximating $\rho^{(2)}\left(\mathbf{r}, \theta, \mathbf{r}, \theta^{\prime}\right)$ by $\rho(\mathbf{r}, \theta) \rho\left(\mathbf{r}, \theta^{\prime}\right)$. Within this approximation, the Fourier transform of Eq. (A7) with respect to the angular variable, $\theta$, leads to

$$
\begin{aligned}
\partial_{t} \hat{\rho}_{n}(\mathbf{r})= & -\epsilon n^{2} \hat{\rho}_{n}(\mathbf{r})-u\left[\partial_{x}\left(\hat{\rho}_{n+1}(\mathbf{r})+\hat{\rho}_{n-1}(\mathbf{r})\right)\right. \\
& \left.+\mathrm{i} \partial_{y}\left(\hat{\rho}_{n-1}(\mathbf{r})-\hat{\rho}_{n+1}(\mathbf{r})\right)\right] \\
& -\epsilon \gamma n\left[\hat{\rho}_{-1}(\mathbf{r}) \hat{\rho}_{n+1}(\mathbf{r})-\hat{\rho}_{1}(\mathbf{r}) \hat{\rho}_{n-1}(\mathbf{r})\right](\mathrm{A} g)
\end{aligned}
$$

where $\rho(\mathbf{r}, \theta)=\sum_{n=-\infty}^{\infty} \hat{\rho}_{n}(\mathbf{r}) \mathrm{e}^{-\mathrm{i} n \theta}$ and $\hat{\rho}_{n}(\mathbf{r})$ is complex. In 22], we have argued that at the onset of collective motion, only the lower modes are important. Therefore, we truncate the above set of infinitely many equations by ignoring all $\hat{\rho}_{n}$ such that $n>1$. Furthermore, if we pick the initial angular perturbation to be directed towards the positive $x$ direction, then we need only consider the real part of $\hat{\rho}_{ \pm 1}$, as the imaginary part of $\hat{\rho}_{ \pm 1}$ will never be excited [22]. With these simplifications, the original single particle density function is approximated as

$$
\rho(\mathbf{r}, \theta) \simeq \alpha(\mathbf{r})+2 \beta(\mathbf{r}) \cos \theta,
$$

where $\alpha(\mathbf{r})$ corresponds to the local density, and $\beta(\mathbf{r})$ corresponds to the local vectorial order parameter since

$$
\langle\mathbf{v}(\mathbf{r})\rangle=\langle(\cos \theta, \sin \theta)\rangle_{\mathbf{r}}=(\beta(\mathbf{r}), 0) .
$$

From Eq. (A9), the reduced model for the onset of collective motion is therefore described by the following equations:

$$
\partial_{t} \alpha=-2 u \partial_{x} \beta \quad, \quad \partial_{t} \beta=-u \partial_{x} \alpha+\epsilon(\gamma \alpha \beta-\beta) .
$$

\section{Appendix B: Non-secular terms}

We consider here the non-secular terms in $\alpha, \beta$ (c.f. Eqs (15) and (17)). Writing $\alpha, \beta$ in the renormalized forms as follow:

$$
\begin{aligned}
& \alpha=1+\sqrt{2}\left(B_{-} f_{-}-B_{+} f_{+}\right)+\frac{\epsilon}{\sqrt{2}} h+\mathcal{O}\left(\epsilon^{2}\right) \\
& \beta=B_{-} f_{-}+B_{+} f_{+}+\frac{\epsilon}{2} g+\mathcal{O}\left(\epsilon^{2}\right)
\end{aligned}
$$

where $h, g$ are functions of $x, t$ to be determined by Eqs (11). We note that $\partial_{t}^{n} B(t)=\mathcal{O}\left(\epsilon^{n}\right)$, and so to order $\epsilon$, we have from Eqs (11) the following differential equations governing $h, g$ :

$$
\begin{aligned}
0= & \sqrt{2}\left(E_{-} f_{-}-E_{+} f_{+}\right)+\frac{1}{\sqrt{2}} \partial_{t} h+\frac{1}{\sqrt{2}} \partial_{x} g \\
0= & E_{-} f_{-}-E_{+} f_{+}+\frac{1}{2} \partial_{t} g+\frac{1}{2} \partial_{x} h \\
& -\epsilon\left[(\gamma-1)\left(B_{-} f_{-}+B_{+} f_{+}\right)+\sqrt{2}\left(B_{-}^{2} f_{-}-B_{+}^{2} f_{+}\right)\right]
\end{aligned}
$$

where $E_{ \pm} \equiv \epsilon^{-1} \partial_{t} B_{ \pm}$. Similar to the derivation of Eq. (14), the above equations can be combined to give the following second order differential equation for $h$ :

$$
\begin{aligned}
\left(\partial_{x}^{2}-\partial_{t}^{2}\right) h= & 4\left(E_{-} f_{-}^{\prime}+E_{+} f_{+}^{\prime}\right) \\
& +2 \epsilon\left[(\gamma-1)\left(B_{-} f_{-}^{\prime}+B_{+} f_{+}^{\prime}\right)\right. \\
& \left.+2\left(B_{-}^{2} f_{-}^{\prime}-B_{+}^{2} f_{+}^{\prime}\right)\right] \\
= & 0
\end{aligned}
$$

where the last identity follows from the definition of $E_{ \pm}$ (c.f. Eqs (39) and (40)). Given that $h(x, t=0)=0$ and $\partial_{t} h(x, t=0)=-2\left(E_{-} f_{-}-E_{+} f_{+}\right)$(c.f. Eq. (B3) $)$, we have

$$
\begin{aligned}
h(x, t) & =2 \int_{x-t}^{x+t}\left[E_{+}(0)-E_{-}(0)\right] f(s) \mathrm{d} s \\
& =-2 b^{2} \gamma \int_{x-t}^{x+t} f(s) \mathrm{d} s \\
& =\gamma\left(h_{-}-h_{+}\right),
\end{aligned}
$$

where $f$ is as defined in Eq. (29), and $h_{ \pm}$are as defined in Eqs (21). Moreover, given the expression for $h$, we can now employ Eq. (B3) to deduce that

$$
g(x, t)=(1-\gamma)\left(g_{-}-g_{+}\right),
$$

where $g_{ \pm}$are also defined in Eqs (21).

\section{Acknowledgments}

The author thanks Fernando Peruani and Frank Jülicher for helpful discussions.
[1] J. Toner, Y. Tu, and S. Ramaswamy, Annals of Physics 318, 170 (2005).
[2] I. D. Couzin, J. Krause, N. R. Franks, and S. A. Levin, Nature 433, 513 (2005). 
[3] J. Buhl, D. J. T. Sumpter, I. D. Couzin, J. J. Hale, E. Despland, E. R. Miller, and S. J. Simpson, Science 312, 1402 (2006).

[4] D. J. T. Sumpter, Philosophical Transactions of the Royal Society B: Biological Sciences 361, 5 (2006).

[5] T. Vicsek and A. Zafiris, e-print arXiv:1010.5017.

[6] L. Tsimring, H. Levine, I. Aranson, E. Ben-Jacob, I. Cohen, O. Shochet, and W. N. Reynolds, Physical Review Letters 75, 1859 (1995).

[7] I. H. Riedel, K. Kruse, and J. Howard, Science 309, 300 (2005).

[8] E. O. Budrene and H. C. Berg, Nature 349, 630 (1991).

[9] T. Vicsek, A. Czirók, E. Ben-Jacob, I. Cohen, and O. Shochet, Physical Review Letters 75, 1226 (1995).

[10] J. Toner and Y. Tu, Physical Review Letters 75, 4326 (1995).

[11] J. Toner and Y. Tu, Physical Review E 58, 4828 (1998).

[12] S. Ramaswamy, R. Aditi Simha, and J. Toner, EPL 62, 196 (2003).

[13] G. Grégoire and H. Chaté, Physical Review Letters 92, 025702 (2004).

[14] V. Dossetti, F. J. Sevilla, and V. M. Kenkre, Physical Review E 79, 051115 (2009).

[15] P. Romanczuk, I. D. Couzin, and L. Schimansky-Geier, Physical Review Letters 102, 010602 (2009).

[16] M. Aldana, V. Dossetti, C. Huepe, V. M. Kenkre, and H. Larralde, Physical Review Letters 98, 095702 (2007).

[17] M. R. D’Orsogna, Y. L. Chuang, A. L. Bertozzi, and L. S. Chayes, Physical Review Letters 96, 104302 (2006).

[18] K. Kruse, J. F. Joanny, F. Jülicher, J. Prost, and K. Sekimoto, Physical Review Letters 92, 078101 (2004).

[19] E. Bertin, M. Droz, and G. Grégoire, Physical Review E 74, 022101 (2006).

[20] F. Peruani, A. Deutsch, and M. Bär, The European Physical Journal - Special Topics 157, 111 (2008).
[21] E. Bertin, M. Droz, and G. Gregoire, Journal of Physics A: Mathematical and Theoretical 42, 445001 (2009).

[22] C. F. Lee, Physical Review E 81, 031125 (2010).

[23] M. H. Holmes, Introduction to Perturbation Methods (Springer, 1995).

[24] C. M. Bender and S. A. Orszag, Advanced Mathematical Methods for Scientists and Engineers: Asymptotic Methods and Perturbation Theory (Springer, 1999).

[25] L. Y. Chen, N. Goldenfeld, and Y. Oono, Physical Review Letters 73, 1311 (1994).

[26] L. Y. Chen, N. Goldenfeld, and Y. Oono, Physical Review E 54, 376 (1996).

[27] K. I. Matsuba and K. Nozaki, Physical Review E 56, R4926 (1997).

[28] S. I. Ei, K. Fujii, and T. Kunihiro, Annals of Physics 280, 236 (2000).

[29] K. Nozaki and Y. Oono, Physical Review E 63, 046101 (2001).

[30] E. Kirkinis, Physical Review E 78, 032104 (2008).

[31] I. Stakgold, Green's Functions and Boundary Value Problems (Pure and Applied Mathematics) (John Wiley \& Sons Inc, 1979).

[32] Note that the fact that $B_{-}$is allowed to diverge is due to the incompleteness of this reduce model. Specifically, it is due to the truncation of the full set of density functions $\left\{\hat{\rho}_{n}\right\}$ (c.f. Eq. A9). In other words, more higher order modes will have to be incorporated in the consideration as $t$ grows. On the other hand, as argued in [22], we believe that the consideration of such a reduced model is appropriate at the onset of collective motion, and is beneficial for analytical progress.

[33] R. Zwanzig, Nonequilibrium Statistical Mechanics (Oxford University Press, Oxford, 2001). 